\title{
Review: early treatment of acute herpes zoster may prevent or shorten the duration of postherpetic neuralgia
}

Alper BS, Lewis PR. Does treatment of acute herpes zoster prevent or shorten postherpetic neuralgia? A systematic review of the literature.J Fam Pract 2000 Mar;49:255-64.

QUESTION: In patients with acute herpes zoster, do any treatments alter the incidence or duration of postherpetic neuralgia (PHN)?

\section{Data sources}

Studies were identified by searching Medline (1966 to 28 December 1998) and the Cochrane Controlled Trials Register 1998, by reviewing the reference lists of identified articles, and by doing web based searches.

\section{Study selection}

English language studies were selected if they were randomised controlled trials (RCTs) that enrolled patients with acute herpes zoster and that reported on relevant outcome measures (incidence of pain at any time after rash healing or duration of zoster associated pain or PHN).

\section{Data extraction}

Data were extracted independently by 2 reviewers on study methods, patient characteristics, intervention type and duration, length of follow up, number of dropouts, and outcome measures.

\section{Main results}

42 studies met the selection criteria. The results from 4 RCTs $(\mathrm{n}=692)$ and 4 meta-analyses done using these 4 trials indicated that oral acyclovir, $800 \mathrm{mg} 5$ times daily for 7-10 days, may reduce the incidence of pain at 1-3 months (numbers needed to treat [NNTs] from 3 of the RCTs range from 4-8 with no reported CIs). The largest of the 4 RCTs found no benefit with acyclovir.

In investigations of newer antiviral agents, 1 study $(\mathrm{n}=419)$ found that famciclovir reduced the duration of PHN at 6 months more than did placebo (NNT 12). This study found that famciclovir had no effect on PHN incidence. 1 study $(n=1141)$ that compared valaciclovir with acyclovir found that pain persisting for 6 months was lower in the valaciclovir group (19\% v $26 \%$ on acyclovir, $\mathrm{p}=0.02$, NNT 17). No placebo comparison was done in this study.

RCTs evaluating the effectiveness of steroids were heterogeneous. Most studies either showed no benefit or the results were indeterminate.

1 RCT $(n=80)$ investigating the effects of the tricyclic antidepressant amitriptyline found that $25 \mathrm{mg}$ nightly for 90 days starting within 48 hours of rash onset reduced pain incidence at 6 months $(16 \%$ v $35 \%$ on placebo at 6 mo, NNT 6). Acyclovir given by general practitioners was not controlled in this study.

A single trial $(n=50)$ comparing percutaneous electrical nerve stimulation with famciclovir reported a decrease in pain incidence with stimulation at 3 and 6 months, but no statistics were provided.

\section{Conclusion}

Limited evidence from randomised controlled trials shows that early treatment of acute herpes zoster with various drugs or modalities decreases the incidence or duration of postherpetic neuralgia.

\section{COMMENTARY}

A recent epidemiological study showed that persistent PHN is less common than previously thought. ${ }^{1}$ Patients $<50$ years of age in this study had no occurrences of persistent PHN, and fewer than 1 in 10 older patients had continued pain a year after acute herpes zoster. ${ }^{1}$ Nevertheless, most primary care physicians have cared for patients with severe and persistent $\mathrm{PHN}$, and a treatment that effectively prevents such suffering would be worth having.

The review by Alper and Lewis highlights the continued uncertainty about the extent to which the available treatments prevent or alleviate pain. As the authors point out, individual trials have reached conflicting conclusions about the effectiveness of antiviral agents. Although a metaanalysis was not done in this review, Alper and Lewis describe 4 previous meta-analyses that have concluded that acyclovir has at least a modest effect. As they point out, pooling data causes problems because of variations in the way pain was reported and analysed.

Nevertheless, the only meta-analysis that included data from all the available trials found that acyclovir reduced persistent pain by almost $50 \% 6$ months after acute zoster, although the confidence intervals were wide. ${ }^{2}$ Trial evidence also indicates benefit from famciclovir and valaciclovir. The weight of evidence therefore supports giving antiviral agents to patients at higher risk for PHN-that is, patients $>50$ years of age.

One trial included in this review suggested that amitriptyline, in addition to its proven benefits in improving established PHN, may reduce the incidence of PHN when used during an acute zoster episode. ${ }^{3}$ Tricyclic antidepressants are a much less costly treatment than antiviral agents, and in the doses used, side effects are not usually severe. Unfortunately, co-prescribing of antiviral agents was permitted in this trial; therefore, we do not have sufficient evidence to justify amitriptyline without antiviral agents as the initial treatment strategy. Adding it to an antiviral agent is an inexpensive option, and I now offer it routinely to older patients.

Tim Lancaster, MSc, MBBS Radcliffe Infirmary Oxford, UK

1 Helgason S, Sigurdsson J, Gudmundsson S. The clinical course of herpes zoster: a prospective study in primary care. Eur J Gen Pract 1996;2:12-6.

2 Jackson JL, Gibbons R, Meyer G, et al. The effect of treating herpes zoster with oral acyclovir in preventing postherpetic neuralgia. A meta-analysis. Arch Intern Med 1997;157:90912.

3 Volmink J, Lancaster T, Gray S, et al. Treatments for postherpetic neuralgia: a systematic review of randomized controlled trials. Fam Pract 1996;13:84-91. 